Relations industrielles

Industrial Relations

\title{
Economics of Labor Relations, par C.F. Bloom et H.R. Northrup, Fourth Ed., Himewood, Illinois, R.D. Irwin Inc., 1961, pp. 881.
}

\section{Louis-Marie Tremblay}

Volume 16, numéro 4, octobre 1961

URI : https://id.erudit.org/iderudit/1021700ar

DOI : https://doi.org/10.7202/1021700ar

Aller au sommaire du numéro

Éditeur(s)

Département des relations industrielles de l’Université Laval

ISSN

0034-379X (imprimé)

1703-8138 (numérique)

Découvrir la revue

Citer ce compte rendu

Tremblay, L.-M. (1961). Compte rendu de [Economics of Labor Relations, par C.F. Bloom et H.R. Northrup, Fourth Ed., Himewood, Illinois, R.D. Irwin Inc., 1961, pp. 881.] Relations industrielles / Industrial Relations, 16(4), 513-514. https://doi.org/10.7202/1021700ar

Tous droits réservés (C Département des relations industrielles de l’Université Laval, 1961
Ce document est protégé par la loi sur le droit d'auteur. L’utilisation des services d'Érudit (y compris la reproduction) est assujettie à sa politique d'utilisation que vous pouvez consulter en ligne.

https://apropos.erudit.org/fr/usagers/politique-dutilisation/ 
La partie V présente l'aspect sociotechnique du comportement entre groupes. La partie VI met en relation le comportement de l'organisation et l'aspect culturel. La dernière partie envisage le rôle de l'administrateur comme facteur de changement dans l'organisation.

\section{Louis-Marie Tremblay}

Economics of Labor Relations, par G.F. Bloom et H.R. Northrup, Fourth Ed., Himewood, Illinois, R.D. Irwin Inc., 1961 , pp. 881 .

Cette oeuvre de Bloom et Northrup dont la première édition remonte à 1950 est si bien connue qu'il n'est pas nécessaire d'en faire une recension détaillée, car les lecteurs intéressés ou préoccupés par les aspects économiques des relations industrielles en connaissent déjà la grande valeur et la grande utilité. Pour le bénéfice de ces derniers il suffira de souligner ce que la quatrième édition présente comme nouveau et d'en indiquer la topographie générale.

Comme question de fait, les auteurs ont réécrit en entier leur travail. Mais, ce n'est pas une recomposition qui implique des changements dans les postulats de base ou dans les caractéristiques essentielles des éditions précédentes. Il s'agit plutôt d'une remise à date en fonction de données et de statistiques plus récentes ainsi que d'éléments nouveaux tel que le «Landrum-Griffin Act» de 1959. C'est pourquoi les lecteurs retrouveront dans cette quatrième édition beaucoup d'éléments familiers remis à neuf. Mais, ils y trouveront aussi des choses qui n'existaient pas dans les éditions précédentes. Les auteurs ont en effet profité de cette revision pour ajouter des chapitres sur le problème de l'union et de l'inflation ainsi que sur la productivité. Les auteurs ont fait aussi des additions importantes aux questions des bénéfices marginaux, de l'automation et des tendances de la force de travail.

Comme pour les éditions précédentes, les auteurs se sont efforcés de présenter un «test book», facile d'accès et aussi complet que possible. «A major objective has been the integration of economic facts and economic analysis so that the student may acquire not only an awareness of labor problems but also and understanding of conflicting views concerning their causes and pos- sible solutions. Wherever possible the authors have incorporated in their discussion the latest views and approaches to various labor problems which have appeared in recent articles in professionnal journals. Considerable effort has been made to spell out clearly economic principles and the techniques of economic analysis.» Une revue du texte, même rapide, montre que les auteurs ont atteint leur objectif.

La partie I s'efforce de circonscrire de façon générale et forcément limitée le champ des problèmes du travail dans ses dimensions économiques et sociologiques. La partie II porte sur le syndicalisme. Il y a d'abord un assez bon résumé de l'histoire du syndicalisme américain où l'on consacre proportionnellement peut-être trop d'importance à l'aspect politique. Ceci est suivi d'une bonne description des structures syndicales et du gouvernement des unions quoique les questions de sécurité syndicale et de démocratie industrielle sont un peu escamotées. La IIIe partie a aussi une perspective institutionnelle. Intitulée, "Collective Bargaining 》, elle couvre à peu près tout ce champ, en débutant avec le problème de la reconnaissance syndicale et les techniques de négociation collective pour continuer avec le contenu de la convention collective et son aspect jurisprudentiel et se terminer avec une analyse statique de la grève ainsi que du prétendu pouvoir monopolistique des syndicats. La partie IV utilise les instruments d'analyse économique pour illustrer les principaux problèmes d'ordre économique que rencontrent les travailleurs. C'est la partie la plus considérable et la plus technique du volume. On y analyse intensément les topiques suivantes: le marché du travail, l'offre et la demande de travail, la détermination des salaires, l'emploi et le chômage, la productivité ouvrière, l'union en face du cycle économique et finalement, le cas du syndicalisme par rapport à l'inflation. La partie VI est consacrée aux diverses formes de sécurité sociale, pour la vieillesse, le chômage, la maladie et les accidents. Les parties V et VII portent sur le rôle sans cesse grandissant du gouvernement en matière de relations industrielles. En y consacrant deux parties, les auteurs reconnaissent implicitement cette nouvelle dimension dans les relations patronalesouvrières où l'intervention et le contrôle gouvernemental tendent à se substituer graduellement au jeu libre des parties 
sous prétexte de maintenir la paix sociale et de protéger le bien commun. La partie V analyse le rôle du gouvernement dans la détermination des salaires et des heures de travail. Il y a une bonne revue historique de la règlementation statutaire des salaires: lois des salaires minimums, standards des justes salaires, stabilisation employée pendant la deuxième guerre mondiale; ainsi que leur portée et leurs effets. Une heureuse attention est aussi accordée aux aspects économiques ainsi qu'aux facteurs qui affectent la réduction des heures de travail. Dans la partie VII on fait une revue des principales règlementations gouvernementales concernant le syndicalisme. Sur ce, un chapitre porte sur le contrôle des tactiques syndicales, grève, piquetage, etc. Puis, il y a des analyses très élaborées du \& TaftHartley Act » et du \& Landrum-Griffin Act précédées d'une étude malheureusement insuffisante et inadéquate du Wagner Act. Cette partie se termine avec le rôle du gouvernement comme conciliateur dans les conflits industriels. Finalement, la dernière partie s'efforce de souligner les principaux problèmes internes et externes que le mouvement syndical rencontre en 1960.

Donc, voici un manuel remis à neuf qui tient compte non seulement des aspects économiques mais qui accorde aussi une large part aux aspects historiques et règlementaires, pour s'avérer lun des plus complets et des mieux construits dans le domaine.

\section{Louts-Marue Tremblay}

Man at Work, by William Foote Whyte, Homewood, Illinois, R.D. Irwin, Inc., 1961 , pp. 593, $\$ 10.60$.

L'auteur de «Industry and Society 》 et de * Human Relations in the Restaurant Industry » nous livre maintenant la somme de vingt ans d'expérience en matière de relations humaines dans le milieu du travail. «Man at Work »'impose immédiatement comme l'un des meilleurs volumes dans le domaine. A l'encontre de la plupart des « textbooks 》 qui consiste surtout dans un amas de matériel descriptif, «Man at Work 》 présente un heureux alliage entre la description, les cas et l'analyse théorique. L'auteur procède par induction, dans une approche qui concilie et se situe entre les approches traditionnelles adop- tées par les psychologues et les sociologues.

La première partie sert à élaborer les instruments d'analyse. Whyte s'interroge d'abord sur les déterminants des inter-relations sociales dans le milieu du travail. Pour y répondre, il adopte la méthode dite: «théorie de l'interaction », développée initialement par Arensberg et McGregor, méthode basée sur les concepts: sentiments, activités et interactions. Ces derniers sont les résultantes a) des influences sociales et culturelles qui moulent la personnalité, b) des forces impersonnelles qui agissent sur le travailleur dans le milieu du travail, c) des interactions et des activités des autres. Ceci constitue l'essentiel du schème analytique que l'auteur utilise dans les parties subséquentes.

Les parties 2 et 3 analysent les influences respectives de l'environnement socio-économique et de l'environnement technique et physique sur le schème trinaire, plus spécialement sur la formation des sentiments. Les parties 4,5 et 6 traitent de l'organisation formelle. La partie 4 étudie intensivement les relations patronales ouvrières. La partie 5 porte sur les problèmes qui concernent la hiérarchie (le « line 》) et la partie 6 sur ceux reliés aux différents services («staffs »). Tout au cours de ces développements, l'auteur cherche à dégager les facteurs d'intégration des travailleurs dans leur milieu tant verticalement qu'horizontalement en utilisant largement le cas pour fin d'illustration. Toutefois, l'auteur sous-estime l'importance de l'organisation non-formelle à laquelle il ne consacre d'ailleurs aucun chapitre particulier. De même, le traitement intégral de l'aspect analytique des concepts n'entre pas dans les préoccupations de l'auteur. C'est pourquoi, « Man at Work » nous apparaît comme un complément idéal à un volume de caractère analytique comme * Human Relations in Administration 》 de Robert Dubin.

La dernière partie qui vise à une reformulation théorique par rapport à l'individu, au groupe et à l'organisation, sert de pendant à la première partie. Ce n'est pas une théorie en soi. Mais l'auteur pose les premiers jalons vers un effort futur de théorisation plus complète, laquelle sera rendue possible par l'augmentation de notre connaissance et 